\title{
Near-field scattering from red pigment particles: Absorption and spectral dependence
}

\author{
L. E. McNeil ${ }^{\mathrm{a})}$ \\ Department of Physics and Astronomy, University of North Carolina at Chapel Hill, Chapel Hill, \\ North Carolina 27599-3255
}

\author{
A. R. Hanuska and R. H. French \\ DuPont Central Research and Development, Wilmington, Delaware 19880-0356
}

(Received 6 June 2000; accepted for publication 5 November 2000)

\begin{abstract}
When film coatings are made of pigment particles embedded in a transparent resin, the optical characteristics of the resulting film are determined not only by the bulk optical properties of the constituent materials, but also by the spatial distribution of the light scattered from small particles. If the particles are separated by distances comparable to their diameter, as is the case for high particle concentrations or agglomerated systems, the near-field interactions between the radiation fields of the particles can strongly influence the resulting far-field intensity distribution. In this work we have used full-field finite-element solutions of Maxwell's equations to calculate the near- and far-field scattering patterns for single $500 \mathrm{~nm}$ quinacridone spheres and for pairs of particles. We find that the scattered intensity forms a forward-directed plume that extends far beyond the particle surface, especially at short wavelengths and where the absorption is large. This results in near-field interactions between pairs of particles that can either increase or decrease the scattering (both the total scattering and the fraction of the scattered light that is directed into the backward hemisphere), depending on the orientation of the particle pair relative to the direction of the incident light. In some cases, particularly if the particles are aligned along the incident direction, the two spheres can produce a far-field scattering distribution that is approximately that of a single, larger (sometimes much larger) sphere. If the particles are aligned perpendicular to the incident direction, the strength of the scattering per particle volume is roughly the same as for a single particle, but the scattering is more forward directed. These interaction effects occur even though the surface-to-surface separation of the particles is larger than the distance for which a single particle shows significant scattered intensity. These near-field and far-field phenomena are beyond the limitations of single-scattering and independent multiple-scattering approaches, and the near-field interactions can have a significant effect on the scattering of light from films containing such particles, especially if they tend to form oriented clusters. (C) 2001 American Institute of Physics.
\end{abstract}

[DOI: $10.1063 / 1.1336564$ ]

\section{INTRODUCTION}

The subject of the scattering of electromagnetic radiation by particles is an old and wide-ranging one. Its applications include such phenomena as the blue sky and other atmospheric phenomena, ${ }^{1}$ the obscuration of galaxies by interstellar dust, ${ }^{2}$ the curing of photopolymers for stereolithography, ${ }^{3}$ the transparency of fishes ${ }^{4}$ and the human cornea, ${ }^{5}$ the efficiency of phosphors, ${ }^{6}$ and the appearance of reflective display materials, ${ }^{7}$ paint $^{8}{ }^{\text {and }}$ paper. ${ }^{9}$

When film coatings are made of pigment particles embedded in a transparent resin, the optical characteristics of the resulting film are determined not only by the bulk optical properties of the constituent materials, but also by the microstructural arrangement of the particles and the spatial distribution of the light scattered from these small particles. If the particles are separated by distances comparable to their diameter, as is the case for high particle concentrations or ag-

a) Author to whom correspondence should be addressed; electronic mail: mcneil@physics.unc.edu glomerated systems, the near-field interactions between the radiation fields of the particles can strongly influence the resulting far-field intensity distribution.

For single particles of simple shapes, it is possible to calculate analytically the intensity of the scattered light, using approaches such as Mie theory. ${ }^{10}$ If the particles are sufficiently far apart that the scattered radiation from the different particles can be added incoherently, then the scattering can be completely described by this analysis. In many applications of light scattering, in particular in paints, pigments, and coatings, the particles are sufficiently close together that their radiation fields interact. In this case the scattering from one particle is affected by the adjacent particles, and the assumption of independent scatterers no longer holds. Thus, the near-field intensity pattern becomes crucial, because it determines the interaction of the radiation fields of the particles with one another. Models that deal exclusively with the far field then become inadequate to fully characterize the scattered radiation.

Mie theory, though exact for the particular case of a single spherical particle in a nonabsorbing medium, is not 
easily extended to the realistic situation of multiple, irregularly shaped, interacting particles in an absorbing medium. Mie theory is also typically used only to compute the extinction cross section rather than the details of the electric field at all locations in space, and thus it is difficult to extract information about the near-field intensity distributions that are crucial in understanding the interactions of closely spaced particles. To calculate the scattering in such cases, it is necessary to abandon the analytic approach and instead use numerical methods. This can be done with a finiteelement method, in which the volume containing the scatterers and the medium is divided up into discrete regions that are small compared to the wavelength and the particle size. Maxwell's equations are then solved in each region for a given incident wave, using the appropriate optical constants for the material of which the region is composed. This nearfield solution thus obtained can then be extrapolated to the far field by simply allowing it to propagate through a uniform medium. This technique can in principle be used to calculate the scattering from any number of arbitrarily shaped, inhomogeneous, optically anisotropic particles, in any configuration, if the necessary computing power is available.

In previous publications ${ }^{11-13}$ we have applied this technique to individual particles and small clusters of $\mathrm{TiO}_{2}$ in resin and latex in water, at a single wavelength. Here we consider quinacridone pigment particles in a transparent resin, at a range of wavelengths in the visible light range. The pigment and resin are typical of those used for automotive paints. Quinacridone $\left(\mathrm{C}_{20} \mathrm{H}_{12} \mathrm{~N}_{2} \mathrm{O}_{2}\right)$, a nonazo organic pigment, has two strong absorption bands centered at 270 and $550 \mathrm{~nm}$, which color it red in reflection and transmission. The extinction coefficient $k_{\text {particle }}$ is in the range $10^{-2}$ $<k_{\text {particle }}<1$ for all but the longest wavelengths in the range. This is in contrast to our earlier work, in which we examined particles whose absorption was negligible at the wavelength considered. However, as in our previous work, the particle is in the resonant regime, in which the particle diameter is comparable to the wavelength of light in the medium. Specifically, for this system the Mie size parameter $x$ $=2 \pi a n_{\text {medium }} / \lambda$, where $a$ is the diameter of the particle, $\lambda$ is the wavelength of light in vacuum, and $n_{\text {medium }}$ is the index of refraction of the resin at $\lambda$, is in the range $5<x<20$. In this regime the scattering is strong, especially when weighted by the volume of the particle. However, it is also strongly forward directed rather than being isotropic, as would be the case for a very small particle.

In this work we present calculations of the near- and far-field distributions of scattered light from single quinacridone spheres of $500 \mathrm{~nm}$ diameter, embedded in an acrylic resin in the wavelength range $250 \leqslant \lambda \leqslant 850 \mathrm{~nm}$. We performed the calculations for four types of cases: with realistic values for the index of refraction and extinction coefficient of both the particle and the medium, with the absorption of the particle artificially set to zero, with the absorption of the medium artificially set to zero, and with absorption considered in neither the particle nor the medium. These last three cases are of course not physically realizable, since the index of refraction and the extinction coefficient are linked through the Kramers-Kronig relations and cannot be changed independently. However, these nonphysical situations do allow us to examine explicitly the effects of absorption, i.e., the loss of light from the radiation field, independent of effects due to the magnitude of the wavelength relative to the particle diameter.

To probe the interactions between particles that result from the spatial distribution of the scattered intensity, we have also calculated the near- and far-field distributions for pairs of particles in two different configurations: side-byside, with the centers of the two particles along a line perpendicular to the direction of the incident light; and in-line, with the centers of the two particles along a line parallel to the incident direction. The surface-to-surface spacing of the particles in each configuration is $125 \mathrm{~nm}$. Measured in units of the wavelength of the light in the medium, this spacing ranges from $0.73 \lambda / n_{\text {medium }}$ at $\lambda=270 \mathrm{~nm}$ to $0.25 \lambda / n_{\text {medium }}$ at $\lambda=750 \mathrm{~nm}$. If this spacing is taken as the average spacing in a large ensemble of particles, it would correspond to a particle volume concentration (PVC) of $51.2 \%$, which is much higher than that typically used in pigment applications. However, even at much lower concentrations (for which the average interparticle spacing is much larger), in a random arrangement of particles it can be expected that such close approaches will occur. In a system in which agglomeration tends to occur, as is commonly the case for pigments, such close approaches are quite likely and can profoundly affect the optical properties of the resulting film.

\section{CALCULATIONAL TECHNIQUES}

The optical constants used in these calculations were those of bulk quinacridone and of an acrylic resin. The pigment and resin are typical of those used in the manufacture of automotive coatings. The optical constants of the quinacridone as a function of wavelength were kindly provided by Dr. P. Bujard of Ciba Specialty Chemicals. The optical constants of the acrylic resin were obtained from spectroscopic ellipsometry measurements performed by M. F. Lemon of DuPont. The relative refractive index (index contrast) of the particle in the resin $m=n_{\text {particle }} / n_{\text {medium }}$ ranges from approximately 1.3 to 1.8 over the visible wavelength range. For comparison, a typical value for $m$ for $\mathrm{TiO}_{2}$ in resin in the middle of the visible range is 1.8 , and $m=1.15$ for latex in water at $488 \mathrm{~nm}$. The Mie size parameter $x$ ranges from 19.9 to 5.4. The particles are therefore not small compared to the wavelength (in which case Rayleigh theory would apply), nor are they large enough to be in the regime of geometrical optics. They are larger than those considered in our previous calculations, for which $x \approx 3.4$.

The finite element method adopted in this work to compute the electromagnetic radiation scattering of single- and multiple-particle films makes use of the program EMFLEX (Weidlinger Assoc.). It is based upon piecewise solutions of the time-domain form of Maxwell's equations in source-free space given in Eq. (1), where the computational domain is discretized into a finite number of volume elements. In Eq. (1), $E$ is the electric field amplitude, $\epsilon$ is the electric permit- 
tivity of the medium, and $\mu$ is the magnetic permeability of the medium

$$
\nabla^{2} E-\epsilon \mu \frac{\partial^{2} E}{\partial t^{2}}=0 .
$$

The parameters required to construct the finite element models used throughout this study consist of the edge dimensions in a Cartesian coordinate system, the finite element mesh density, the particle positions, the particle shapes, and the wavelength-dependent optical properties of the materials contained in the model. The number of elements in a particular model varies according to the edge dimensions and the mesh density. Typical models contain between 5 and 10 million elements. A plane wave of circularly polarized electromagnetic radiation is propagated through the model in the time domain while the nodal electric and magnetic fields and amplitudes are computed at each time step. The resulting total and scattered intensities are available at each node for every time step, however, only steady-state results are of interest in this work. To obtain the far-field scattered intensities, the near-field scattered intensities are extrapolated onto the surface of a far-field sphere with a radius that is very large compared to the wavelength of the electromagnetic radiation.

Once the far-field scattered electric field and intensities have been extrapolated, macroscopically observable physical quantities such as the scattering cross-section $C_{\text {sca }}$ of the angular distribution of the scattered light can be computed. The scattering cross-section $C_{\text {sca }}$ defined in Eq. (2) is expressed in units of area, where $\theta$ is the scattering angle, $I_{0}$ is the incident radiation intensity, and $I_{\text {sca }}(\theta)$ is the scattered intensity on the surface of the far-field sphere over which the surface integral is performed

$$
C_{\text {sca }}=\frac{1}{I_{0}} \iint_{4 \pi} I_{\text {sca }}(\theta) d \Omega .
$$

The scattering coefficient $S$ is defined as the scattering cross section normalized by the volume of the scattering material in the model. In Eq. (3), the scattering coefficient $S$ is expressed in units of inverse length

$$
S=\frac{C_{\text {sca }}}{V} .
$$

In addition to the finite-element calculations that form the bulk of this work, we have also performed some analytical calculations of far-field scattering from single spheres using Mie theory. In these calculations we have made use of the program MIETAB. ${ }^{14}$

\section{RESULTS}

\section{A. Absorbing quinacridone particle in absorbing resin}

\section{Short-wavelength, high-absorption region $(250<\lambda<370 \mathrm{~nm})$}

In this region the extinction coefficient $k_{\text {particle }}$ of quinacridone ranges from 0.02 to 0.87 , with the maximum absorption occurring at $\lambda=270 \mathrm{~nm}$. The Mie size parameter is in the range $13.2<x<19.9$. An example of the scattering pattern can be seen in Fig. 1(a). As in thin-film structures, interference between waves scattered from different surfaces produces regions of enhanced or diminished intensity at various locations in the scattering pattern. For wavelengths near $250 \mathrm{~nm}$, the most striking aspect of the scattered intensity distribution is the plume of scattered radiation that extends away from the particle in the forward-scattering (downstream) direction. This plume is detached from the particle, with a local minimum in the scattered intensity occurring at the surface of the particle. The maximum in the scattered intensity, which is located approximately $300 \mathrm{~nm}$ from the surface, can be as high as 2.4 times the incident intensity. Clearly an adjacent particle that fell within this plume would have its radiation field strongly influenced by the scattered light from the first particle. This will be illustrated when we consider the two-particle scattering patterns. The plume extends to a distance of approximately $1 \mu \mathrm{m}$ from the surface of the particle and is about $300 \mathrm{~nm}$ wide.

At slightly longer wavelengths the local minimum immediately downstream of the particle becomes more pronounced, and a torus of scattered intensity appears, with the local maximum in the intensity centered at about $15^{\circ}$ from the forward-scattering direction. This torus becomes more pronounced at larger wavelengths, eventually becoming completely detached from the central plume. As the wavelength is further increased, the central local minimum abruptly disappears and the plume becomes connected to the particle. The maximum intensity also abruptly increases so that scattered intensities of more than twice the incident intensity extend as far as $600 \mathrm{~nm}$ from the particle surface.

The two particles in the side-by-side configuration at $\lambda$ $=270 \mathrm{~nm}$ [Fig. 1(b)] demonstrate striking effects of the interaction of their radiation fields. These dramatic variations in the local electric field are characteristic of photonic crystals, in which dielectric particles are arranged in ordered arrays to produce optical stop bands in particular directions. Not only is there significant scattered intensity between the two plumes approximately $310 \mathrm{~nm}$ downstream of the particle surfaces, interference effects produce a wider region of extinction in the center of the pattern, causing the axis of symmetry of each plume to deviate from the $\theta=0$ direction. Approximately $540 \mathrm{~nm}$ downstream of the particle surfaces, the plumes begin to spread toward one another, and at longer wavelengths the scattered light forms a single plume centered between the two particles.

In the in-line configuration [Fig. 1(c)], the maximum intensity occurs at essentially the same location as for the single-particle case. The region between the two particles shows interference fringes due to reflection by the second particle of light scattered from the first. The most striking effect of the addition of the second particle, however, is the extension of the scattering plume to a distance several microns downstream of the particles. Although the intensity in this extended plume is not so large (approximately $80 \%$ of the incident intensity), it is much larger than the scattered intensity outside the plume (which is negligible). Thus, two particles in this in-line configuration can thereby extend their optical influence over a large region downstream. 


\section{Low-absorption, high-scattering region $(370<\lambda$ $<510 \mathrm{~nm}$ )}

In this wavelength range, the basic shape of the scattering pattern remains constant but the maximum scattered intensity changes. An example can be seen in Fig. 1(d). The maximum intensity, now located at the particle surface, rises to 22.9 times the incident intensity at $\lambda=390 \mathrm{~nm}$. At this wavelength the absorption has a minimum at $k=0.006$ and $x=12.4$. At longer wavelengths the maximum intensity declines as the absorption increases.

The two particles in the side-by-side configuration at $\lambda$ $=390 \mathrm{~nm}$ [Fig. 1(e)] show a pattern very similar to that at $270 \mathrm{~nm}$. In the in-line configuration [Fig. 1(f)], essentially all of the scattering is confined to the region between the particles.

\section{High-absorption, moderate-wavelength region ( 510 $<\lambda<610 \mathrm{~nm}$ )}

In this wavelength range the absorption continues to increase, reaching a maximum of $k_{\text {particle }}=0.68$ at $\lambda=560 \mathrm{~nm}$ before declining steeply. The location of the maximum intensity region again begins to shift away from the particle surface, as it did in the high-absorbance region at shorter wavelengths. An example can be seen in Fig. 2(a). Even at a distance of $250 \mathrm{~nm}$ downstream from the surface, the intensity remains above five times the incident value.

The two particles in the side-by-side configuration at $\lambda$ $=560 \mathrm{~nm}$ [Fig. 2(b)] show a pattern that is similar to that for smaller wavelengths, but with some notable differences. The axes of symmetry of the individual plumes now deviate inward from the $\theta=0$ direction. The single centered plume now appears much closer to the surface of the particles, at a distance of $420 \mathrm{~nm}$ downstream.

In the in-line configuration, the scattering pattern is essentially two single-particle plumes stacked on one another. The highest scattered intensity occurs between the particles, as for $390 \mathrm{~nm}$ light, but for the longer wavelength the difference in intensity between the absolute maximum there and the local maximum in the plume is small. The effect of absorption is therefore to distribute the scattered intensity more evenly throughout the volume surrounding the particles.

\section{Long-wavelength, high-scattering region $(610<\lambda<850 \mathrm{~nm})$}

For $\lambda>610 \mathrm{~nm}$ the extinction coefficient $k_{\text {particle }}<0.02$ and absorption plays a negligible role in determining the scattering pattern. In this region the basic shape of the scattered radiation remains the same, but the maximum intensity declines. An example can be seen in Fig. 2(d). At $\lambda$ $=750 \mathrm{~nm}$ the maximum intensity occurs inside the particle, at the $\theta=0$ position near the surface. The scattered intensity extends approximately $250 \mathrm{~nm}$ away from the particle surface.

The two particles in the side-by-side configuration at $\lambda$ $=750 \mathrm{~nm}$ [Fig. 2(e)] show a pattern similar to that seen in Fig. 1(e). Here, however, the centered plume begins only about $170 \mathrm{~nm}$ downstream of the particle surfaces. The maximum scattered intensity (which occurs inside the particles at this wavelength) is very similar to that for a single particle.

In the in-line configuration [Fig. 2(f)], the maximum of the scattered intensity increases, and it occurs in the downstream particle, at roughly the same position as in the singleparticle case. The region between the two particles has very little scattered light.

\section{B. Effects of particle and resin absorption}

The effects of absorption of light by the particle on the distribution of scattered light in the near field (as opposed to its maximum intensity) are significant only in wavelength regions where the extinction coefficient of the quinacridone is larger than approximately 0.02 .

For the short-wavelength, high absorption region (250 $<\lambda<370 \mathrm{~nm}$ ) the case in which the absorption has been "turned off" (but, unrealistically, the index of refraction remains the same), the scattered light is concentrated in a "hot spot" at the surface of the particle, reaching a maximum intensity of 43.7 times the incident intensity. This is a much larger concentration of scattering than was seen ${ }^{11}$ for a 200 $\mathrm{nm} \mathrm{TiO}_{2}$ particle at $560 \mathrm{~nm}$, despite the fact that the index contrast in the quinacridone case (1.47) is smaller than for the $\mathrm{TiO}_{2}$ case (1.81). However, the smaller size parameter $\left(x=18.3\right.$ for quinacridone versus 3.4 for $\mathrm{TiO}_{2}$ ) leads to a much higher scattering cross section $C_{\text {sca }}$ for the quinacridone particle $\left(0.40\right.$ vs $\left.0.06 \mu \mathrm{m}^{2}\right)$, although the volumenormalized scattering coefficient $S=C_{\mathrm{sca}} /\left(\pi a^{3} / 6\right)$ is larger for $\mathrm{TiO}_{2}$.

A qualitative comparison of the absorbing and nonabsorbing cases in this wavelength region shows that the effect of absorption is to produce a plume of scattered intensity at some distance from the particle in the forward-scattering direction. This means that the maximum in the intensity of the light scattered from the particle occurs at $250 \mathrm{~nm}$ or more away from the particle.

In the absorption band that occurs at longer wavelengths $(510<\lambda<610 \mathrm{~nm})$, the "removal" of absorption from the particle does not change the shape of the scattering pattern significantly. However, the intensity maximum, located at the particle surface at $\theta=0$, is as much as ten times larger for the nonabsorbing particle than for the absorbing one. The effect of absorption in the particle in this region is therefore primarily to suppress the scattered intensity, rather than to change its spatial distribution. This is in contrast to the shorter-wavelength region, in which the presence of absorption strongly modifies the scattering pattern.

The extinction coefficient of the acrylic resin used in these calculations is small for most of the wavelength range considered. It rises above $10^{-3}$ only for wavelengths $\lambda$ $<320 \mathrm{~nm}$, and even at $\lambda=250 \mathrm{~nm}, k_{\text {medium }}$ is only 8 $\times 10^{-3}$. The effects of absorption in the medium are therefore only noticeable at the shortest wavelengths, where they reduce the intensity of the scattered light in the high-intensity region by about $10 \%$. 


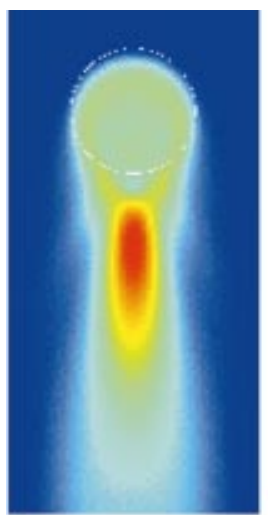

(a)

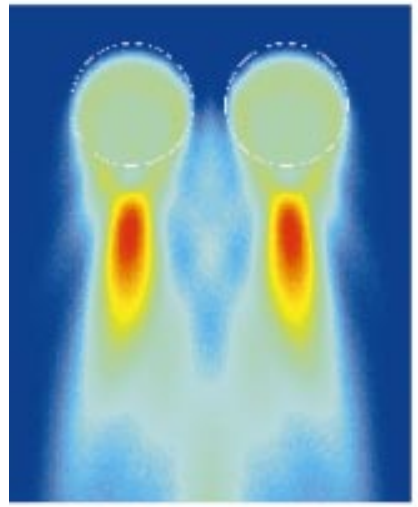

(b)

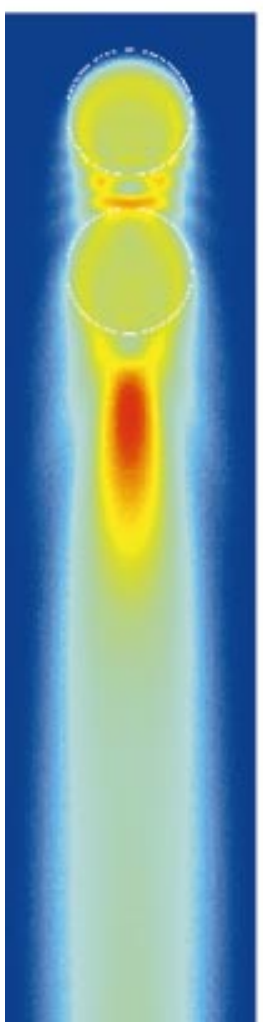

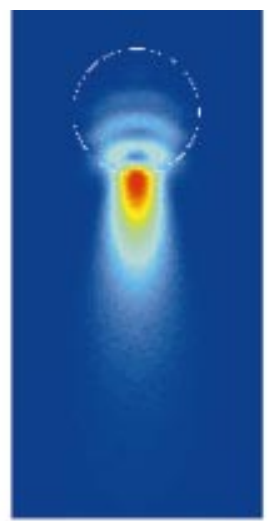

(d)

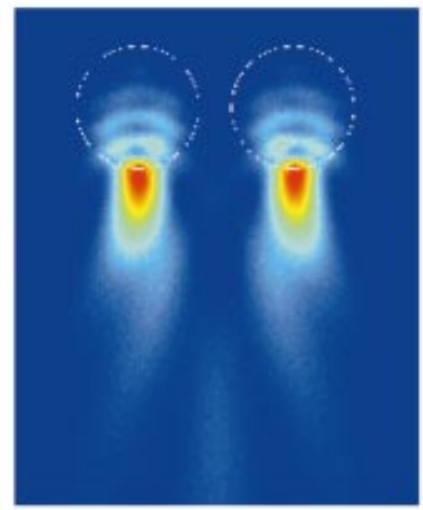

(e)

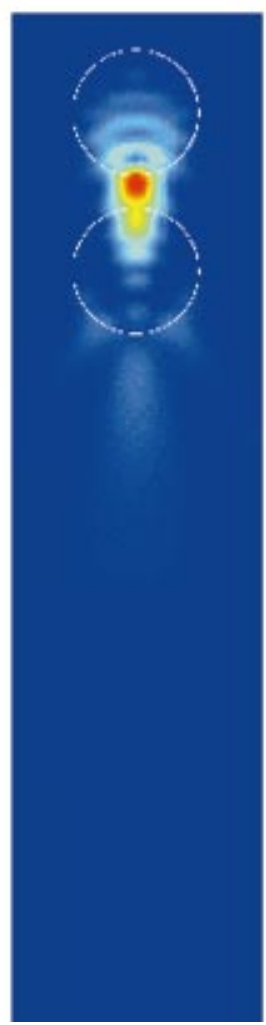

FIG. 1. (Color) Scattered intensity for absorbing particles in absorbing resin. All intensity scales in units of incident intensity. (a) Single particle, $\lambda$ $=270 \mathrm{~nm}$. Spatial scale $1 \mu \mathrm{m} \times 2 \mu \mathrm{m}$, intensity scale 2.17 (red) $-2.32 \times 10^{-3}$ (blue). (b) Two particles in the side-by-side configuration, $\lambda=270 \mathrm{~nm}$. Spatial scale $2 \mu \mathrm{m} \times 2 \mu \mathrm{m}$, intensity scale 2.22 (red) $-1.83 \times 10^{-3}$ (blue). (c) Two particles in the in-line configuration, $\lambda=270 \mathrm{~nm}$. Spatial scale $1 \mu \mathrm{m} \times 5 \mu \mathrm{m}$, intensity scale 1.81 (red) $-2.20 \times 10^{-3}$ (blue) (d) Single particle, $\lambda=390 \mathrm{~nm}$. Spatial scale $1 \mu \mathrm{m} \times 2 \mu \mathrm{m}$, intensity scale 23.4 (red) $-4.59 \times 10^{-3}$ (blue). (e) Two particles in the side-by-side configuration, $\lambda=390 \mathrm{~nm}$. Spatial scale $2 \mu \mathrm{m} \times 2 \mu \mathrm{m}$, intensity scale 22.8 (red) $-5.97 \times 10^{-3}$ (blue). (f) Two particles in the in-line configuration, $\lambda=390 \mathrm{~nm}$. Spatial scale $1 \mu \mathrm{m} \times 5 \mu \mathrm{m}$, intensity scale 23.4 (red) $-2.49 \times 10^{-3}$ (blue). 


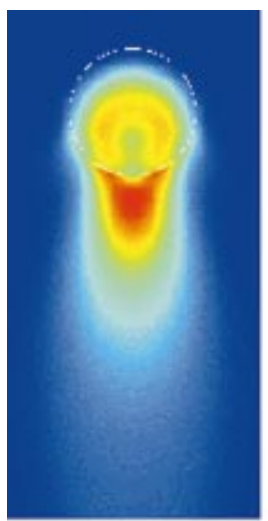

(a)

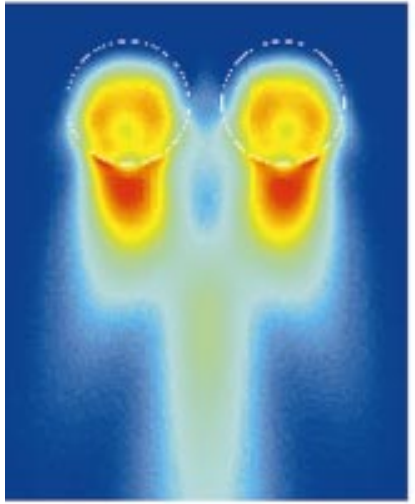

(b)

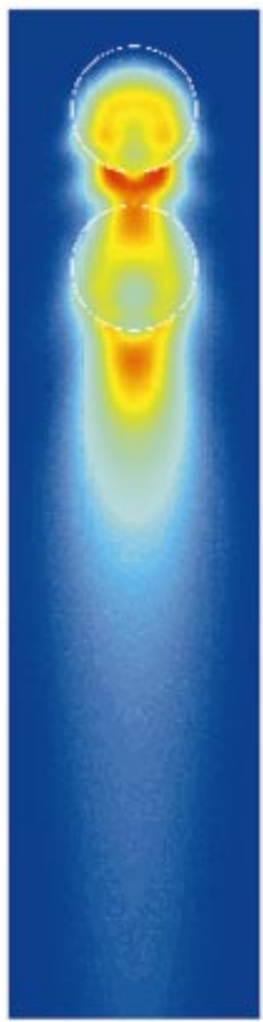

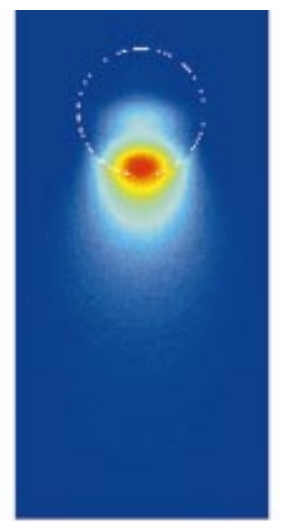

(d)

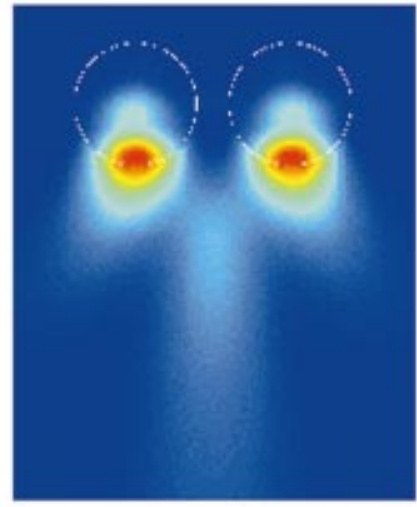

(e)

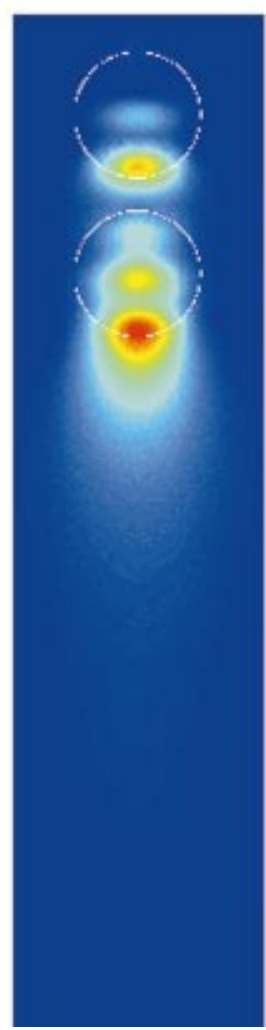

FIG. 2. (Color) Scattered intensity for absorbing particles in absorbing resin. All intensity scales in units of incident intensity. (a) Single particle, $\lambda$ $=560 \mathrm{~nm}$. Spatial scale $1 \mu \mathrm{m} \times 2 \mu \mathrm{m}$, intensity scale 2.07 (red) $-3.90 \times 10^{-3}$ (blue). (b) Two particles in the side-by-side configuration, $\lambda=560 \mathrm{~nm}$. Spatial scale $2 \mu \mathrm{m} \times 2 \mu \mathrm{m}$, intensity scale 1.93 (red) $-3.04 \times 10^{-3}$ (blue). (c) Two particles in the in-line configuration, $\lambda=560 \mathrm{~nm}$. Spatial scale $1 \mu \mathrm{m} \times 5 \mu \mathrm{m}$, intensity scale 2.07 (red) $-4.05 \times 10^{-3}$ (blue) (d) Single particle, $\lambda=750 \mathrm{~nm}$. Spatial scale $1 \mu \mathrm{m} \times 2 \mu \mathrm{m}$, intensity scale 10.4 (red) $-4.76 \times 10^{-3}$ (blue). (e) Two particles in the side-by-side configuration, $\lambda=750 \mathrm{~nm}$. Spatial scale $2 \mu \mathrm{m} \times 2 \mu \mathrm{m}$, intensity scale 10.2 (red) $-2.75 \times 10^{-3}$ (blue). (f) Two particles in the in-line configuration, $\lambda=750 \mathrm{~nm}$. Spatial scale $1 \mu \mathrm{m} \times 5 \mu \mathrm{m}$, intensity scale 16.5 (red) $-4.37 \times 10^{-3}$ (blue). 
TABLE I. Far-field scattering parameters for single quinacridone particle, two particles in the in-line configuration, and two particles in the side-byside configuration. $B$ is the backscattering fraction; $C_{\text {sca }}$ is the scattering cross section; $S$ is the scattering cross section divided by the volume of the particle; and $R$ is the calculated pseudoreflectance.

\begin{tabular}{|c|c|c|c|c|c|}
\hline Wavelength & Absorption & & $\begin{array}{l}\text { Single } \\
\text { particle }\end{array}$ & In-line & Side-by-side \\
\hline \multirow[t]{4}{*}{$270 \mathrm{~nm}$} & 0.873 & $B$ & $3.67 \%$ & $1.95 \%$ & $2.70 \%$ \\
\hline & & $C_{\mathrm{sca}}\left(\mu \mathrm{m}^{2}\right)$ & 0.236 & 0.418 & 0.532 \\
\hline & & $S\left(\mu \mathrm{m}^{-1}\right)$ & 3.606 & 3.193 & 4.064 \\
\hline & & $R$ & $2.23 \%$ & $0.99 \%$ & $1.96 \%$ \\
\hline \multirow[t]{4}{*}{$390 \mathrm{~nm}$} & 0.006 & $B$ & $6.87 \%$ & $12.51 \%$ & $6.65 \%$ \\
\hline & & $C_{\mathrm{sca}}\left(\mu \mathrm{m}^{2}\right)$ & 0.691 & 0.684 & 1.324 \\
\hline & & $S\left(\mu \mathrm{m}^{-1}\right)$ & 10.558 & 5.225 & 10.115 \\
\hline & & $R$ & $23.43 \%$ & $20.46 \%$ & $21.70 \%$ \\
\hline \multirow[t]{4}{*}{$560 \mathrm{~nm}$} & 0.677 & $B$ & $4.63 \%$ & $4.87 \%$ & $4.22 \%$ \\
\hline & & $C_{\mathrm{sca}}\left(\mu \mathrm{m}^{2}\right)$ & 0.245 & 0.332 & 0.498 \\
\hline & & $S\left(\mu \mathrm{m}^{-1}\right)$ & 3.743 & 2.536 & 3.804 \\
\hline & & $R$ & $2.70 \%$ & $1.54 \%$ & $2.52 \%$ \\
\hline \multirow[t]{4}{*}{$750 \mathrm{~nm}$} & 0.000 & $B$ & $7.37 \%$ & $8.37 \%$ & $6.76 \%$ \\
\hline & & $C_{\mathrm{sca}}\left(\mu \mathrm{m}^{2}\right)$ & 0.683 & 0.415 & 1.349 \\
\hline & & $S\left(\mu \mathrm{m}^{-1}\right)$ & 10.435 & 1.176 & 10.306 \\
\hline & & $R$ & $25.64 \%$ & $3.28 \%$ & $23.22 \%$ \\
\hline
\end{tabular}

\section{Far-field scattering}

The far-field scattering patterns are, as expected, much simpler than the near field. For an isotropic sphere, the farfield pattern for a single particle can be calculated analytically, using Mie theory. The angular distribution of the scattered radiation obtained here from the EMFLEX calculations agrees with the analytical calculation to within the accuracy of the finite element model (which for the number of elements used in these calculations is approximately $3 \%$ ). ${ }^{13}$ The far-field radiation is strongly forward directed because the size of the particle is comparable to the wavelength of the light. The half angle of the forward cone containing $50 \%$ of the scattered radiation ranges from $8.5^{\circ}$ at $\lambda=250 \mathrm{~nm} 31.5^{\circ}$ at $850 \mathrm{~nm}$. The backscattered portion of the radiation $B$, (the fraction of the scattered light with scattering angles $\theta>90^{\circ}$ ), which is most relevant for applications in pigments and coatings, never exceeds the $14 \%$ reached for $610 \mathrm{~nm}$ light. The values of $B$ as well as $C_{\text {sca }}$ and $S$ obtained from the EMFLEX calculations are shown in Table I for a single particle and for two particles in the in-line and side-by-side configurations.

For the single particle the parameters have the same trend with wavelength, being larger at wavelengths where the absorption is low and smaller where the absorption is high. Comparison of $B$ for wavelengths at which the absorption is similar shows that for a single particle the scattering tends to become less anisotropic as the wavelength becomes larger relative to the particle size.

When two particles are present, the far-field parameters depend on the configuration, and in a rather complex way. If the particles were far enough apart to be considered single scatterers, the total $C_{\text {sca }}$ would simply be proportional to the number of particles, and $S$ and $B$ would be the same as for a single particle. At wavelengths where the absorption is high, this is approximately the case. However, if the two particles are in the in-line configuration, $S$ decreases, an effect more pronounced at $560 \mathrm{~nm}$ than at $270 \mathrm{~nm}$. This might be ex- pected since the second particle lies in the shadow of the first, and the first particle's absorption prevents some of the light from reaching the second particle and being scattered by it. In the side-by-side configuration the interaction of the particles enhances their scattering and $S$ is increased over the single-particle value. In both cases the interaction of the particles modifies the scattering from that expected in a singlescattering picture.

At wavelengths where the absorption is negligible (390 and $750 \mathrm{~nm}$ ), the side-by-side configuration produces a roughly constant $S$. The interaction of the radiation fields of the two particles therefore does not enhance the scattering when absorption is not present. For the in-line configuration, at $390 \mathrm{~nm} C_{\text {sca }}$ is almost unaffected by the addition of the second particle, whereas at $750 \mathrm{~nm} C_{\text {sca }}$ actually decreases when the second particle is added. This behavior cannot be explained in an independent multiple-scattering picture for nonabsorbing particles, in which light scattered from one particle either continues to the far field or is scattered a second time by the other particle.

\section{DISCUSSION}

\section{A. Near-field interactions}

The most striking characteristic of these scattering patterns is the long scattering plume, which extends the particle's influence far away from its surface, especially when the absorption is strong and the wavelength is short. This plume means that the addition of a second particle may either increase the fraction of the light that is backscattered or decrease it, depending on the location of the second particle relative to the direction of the incident light. Statistically averaged calculations over random microstructures will obviously fail to capture the true nature of the near-field interaction. Since the purpose of a pigmented coating is to produce backscattering of incident light, the details of this nearfield interaction and its effect on the fraction of the incident light scattered into the backward hemisphere are quite important in applications. In a random array of particles, in-line and side-by-side configurations are equally likely. However, if the processing of a film tends to produce clusters of pigment particles with a preferred orientation, one configuration may predominate. In a photonic crystal, in which the particles are deliberately placed in an ordered array, these nearfield effects form the basis of the phenomenon of optical stop bands.

If more than one particle is present, then each particle will be excited by a field that is the sum of the incident field and the scattered fields of the other particles. The particles can be considered independent if they are far enough apart that the scattered field from the two particles is simply the sum of the scattered fields that would be present due to the individual particles. This is clearly not the case for the spacings considered here. Even for the side-by-side configuration, where for the single particle the scattered intensity 125 $\mathrm{nm}$ away from the surface in a direction perpendicular to the incident direction is negligible, the presence of the second particle modifies the scattering pattern in regions far from the particle surfaces. This can be seen most clearly in the appear- 
ance of scattered intensity along the line joining the particle centers, especially at $\lambda=560 \mathrm{~nm}$. Similar results have been seen for two $\mathrm{TiO}_{2}$ particles in close proximity. ${ }^{11,13}$

\section{B. The far field}

In the far field, $C_{\mathrm{sca}}$ measures the strength of the scattering, and for a single particle it depends in a complicated way on the particle size and optical constants (index of refraction and extinction coefficient), the optical constants of the medium in which the particle is embedded, and the wavelength of the light. The volume-normalized parameter $S$ is of even more direct relevance to applications in pigments and coatings, since a system with a larger $S$ can produce the same degree of opacity or "hiding" with a smaller quantity of pigment. In previous studies of clusters of seven particles in the high-contrast system of $200 \mathrm{~nm} \mathrm{TiO}_{2}$ spheres in resin ${ }^{13}$ we observed that the effects of particle interaction on the volume-normalized scattering parameter $S$ became significant when the particles had a surface-to-surface separation $L$ of less than $200 \mathrm{~nm}$ for illumination at $\lambda=560 \mathrm{~nm}$. This corresponded to a separation measured in wavelengths of $L_{\lambda}=n_{\text {medium }} L / \lambda=0.5$. Essentially no change in $S$ was observed for separations $L_{\lambda} \geqslant 0.8$. Similar results were obtained for pairs of particles of more realistic shapes. These calculations were performed for random illumination direction, and thus included both in-line and side-by-side configurations. For the quinacridone-resin system at the surface-to-surface spacing $L=125 \mathrm{~nm}$ considered here, we have values of $L_{\lambda}$ from 0.7 at short wavelengths to 0.2 at long wavelengths. Based on our findings in $\mathrm{TiO}_{2}$, we would expect that interaction between the radiation fields of the particles would have a significant effect on the scattering parameter, and that this effect would be more pronounced for shorter wavelengths. As we shall see, the presence of absorption complicates the situation considerably.

However, some insight can be gained by comparing the scattering of the two particles to that of a single, larger particle. The diameter of the equivalent single particle (i.e., the single particle that gives the same $S$ ) can then be calculated using Mie theory. The diameter at which $S$ has its maximum for a given wavelength is less than or equal to $500 \mathrm{~nm}$ for the wavelengths considered here, so increasing the particle size decreases the volume-normalized single-particle scattering. This is similar to the case of the $\mathrm{TiO}_{2}$ particles considered previously, ${ }^{11}$ for which the $200 \mathrm{~nm}$ diameter produced the maximum $S$ for a single particle at the wavelength for which the calculation was done. The diameters $d_{\text {equ }}$ of the spheres that give the same value of $S$ for the various wavelengths are shown in Table II, together with the fraction of the incident light that would be scattered into the backward hemisphere by a single sphere of that diameter.

A sphere with volume equivalent to that of two $500 \mathrm{~nm}$ spheres would have a diameter of $630 \mathrm{~nm}$. This is almost exactly the diameter of the single sphere that gives the same $S$ as the two in-line spheres under $390 \mathrm{~nm}$ illumination, but at longer wavelengths the two particles give an $S$ corresponding to a single particle that is considerably larger. The interaction of the two particles therefore encompasses a volume
TABLE II. Diameter of sphere with $S$ value equal to that of two $500 \mathrm{~nm}$ spheres in the in-line configuration, and fraction of light scattered into the backward hemisphere for a sphere of that diameter. Note that a sphere of volume equal to that of the two $500 \mathrm{~nm}$ spheres would have a diameter of $630 \mathrm{~nm}$.

\begin{tabular}{ccc}
\hline \hline Wavelength & $d_{\text {equ }}(\mathrm{nm})$ & $\begin{array}{c}\text { Equivalent } B \\
(\%)\end{array}$ \\
\hline 270 & 566 & 3.64 \\
390 & 633 & 11.55 \\
560 & 724 & 4.20 \\
750 & 3000 & 7.53 \\
\hline \hline
\end{tabular}

that is considerably larger than the actual volume of the particles.

The behavior of the anisotropy of the scattering, i.e., the backscattering fraction, is less straightforward. In a multiplescattering picture, in which the total $C_{\mathrm{sca}}$ is enhanced by light scattered from one particle being scattered a second time by a second particle, the strongly forward-directed character of the single-particle pattern would be modified by the presence of the second particle, so that the portion of the light scattered at angles away from the illumination direction would be expected to be larger for two particles than for one. This effect is well known in the case of pigments, in which multiple scattering produces significant diffuse reflectance even if the single-particle scattering is strongly forward directed. However, two particles in the side-by-side configuration actually scatter a smaller fraction of the incident light into the backward hemisphere than does a single particle, especially at the shortest wavelength. Here the interaction of the radiation fields of the two particles produces a scattering pattern that is more strongly forward directed than that of a single particle. Such a scattering pattern would be characteristic of a larger particle.

For the in-line configuration the picture is different. Except at $270 \mathrm{~nm}$, the presence of the second particle increases the fraction of the scattered light that is directed into the backward hemisphere, as would be expected in a multiplescattering picture. The values obtained are quite similar to those for the equivalent- $S$ sphere (sphere of diameter $d_{\text {equ }}$ ), as shown in Table II. However, for the short-wavelength case, where the surface of the second particle is located where the maximum of the scattered intensity for the first particle would otherwise occur, the effect of the second particle is to reduce the backscattering. Thus, in effect, the two particles together produce a scattering pattern that is more strongly forward directed than would either one alone, and than they would if combined into a single sphere.

In order to relate these findings to measurable optical properties of real films, we have calculated a pseudoreflectance $R$ for each case (with the results shown in Table I). This quantity should behave similarly to the measured diffuse reflectance. It is formed from the backscattering fraction $B$, multiplied by the scattering efficiency $C_{\text {sca }} / p \pi(a / 2)^{2}$ (where $a$ is the particle diameter and $p$ is the number of particles, either 1 or 2) to account for the variation of the total scattering with wavelength. This is in turn multiplied by the albedo $C_{\text {sca }} / C_{\text {ext }}$ to account for absorption. The resulting pseudoreflectance $R$ is thus 


$$
R=B \frac{C_{\mathrm{sca}}}{p \pi(a / 2)^{2}} \frac{C_{\mathrm{sca}}}{C_{\mathrm{ext}}} .
$$

Not surprisingly, the greatest reflectance occurs at wavelengths for which the absorption is smallest, so that the light has the greatest probability of being scattered back rather than absorbed. The orientation of the particles plays a significant role in such cases, as is demonstrated by the wide variation in $R$ at $750 \mathrm{~nm}$ for different orientations. While such a calculation would not be expected to reproduce quantitatively the measured reflectance of a real film, the variation in $R$ with wavelength shown here is qualitatively similar to what we have measured ${ }^{15}$ in real quinacridone films for PVCs of $\sim 14 \%$ or less.

\section{CONCLUSIONS}

In this work we have used full-field finite-element solutions to Maxwell's equations to calculate the near- and farfield scattering patterns for single $500 \mathrm{~nm}$ quinacridone spheres and for pairs of particles. We find that the scattered intensity forms a forward-directed plume that extends far beyond the particle surface, especially at short wavelengths and where the absorption is large. This results in near-field interactions between pairs of particles that can either increase or decrease the scattering (both the total scattering and the fraction of the scattered light that is directed into the backward hemisphere), depending on the orientation of the particle pair relative to the direction of the incident light. This nonmonotonic behavior cannot be extracted from an independent multiple-scattering picture, nor from a statistically averaged calculation over a random microstructure. This anisotropy in the effects of particle interactions is also the basis for the formation of optical stop bands in photonic crystals. In some cases, particularly if the particles are aligned along the incident direction, the two spheres can produce a far-field scattering distribution that is approximately that of a single, larger (sometimes much larger) sphere. If the particles are aligned perpendicular to the incident direction, the strength of the scattering per particle volume is roughly the same as for a single particle, but the scattering is actually more forward directed, rather than less so as would be expected from a multiple-scattering approach. These interaction effects occur even though the surface-to-surface separation of the particles is larger than the distance for which a single particle shows significant scattered intensity. Such near-field interactions can have a significant effect on the scattering of light from films containing such particles, especially if they tend to form oriented clusters.

\section{ACKNOWLEDGMENTS}

The authors wish to thank J. Mould, Jr., for computational support in implementing the finite element code and A. Miller for assistance with the Mie scattering calculations. The authors are also grateful to P. Bujard for providing the optical constants of quinacridone and to M. F. Lemon for ellipsometry measurements of the resin.

${ }^{1}$ C. F. Bohren, Am. J. Phys. 55, 524 (1987).

${ }^{2}$ S. Chandrasekhar, Radiative Transfer (Dover, New York, 1960).

${ }^{3}$ H. M. Liao and T. W. Coyle, J. Can. Ceram. Soc. 65, 254 (1996).

${ }^{4}$ S. Johnsen and E. A. Widder, J. Theor. Biol. 199, 181 (1999).

${ }^{5}$ G. B. Benedek, Appl. Opt. 10, 459 (1971).

${ }^{6}$ A. Konrad, J. Almanstötter, J. Reichardt, A. Gahn, R. Tidecks, and K. Samwer, J. Appl. Phys. 85, 1796 (1999).

${ }^{7}$ R. Barchini, J. G. Gordon II, and M. W. Hart, Jpn. J. Appl. Phys., Part 1 37, 6662 (1998).

${ }^{8}$ D. G. Phillips and F. W. Billmeyer, Jr., J. Coat. Technol. 48, 30 (1976).

${ }^{9}$ R. W. Johnson, E. S. Thiele, and R. H. French, TAPPI J. 80, 233 (1997).

${ }^{10}$ C. F. Bohren and D. R. Huffman, Absorption and Scattering of Light by Small Particles (Wiley, New York, 1983).

${ }^{11}$ E. S. Thiele and R. H. French, J. Am. Ceram. Soc. 81, 469 (1998).

${ }^{12}$ E. S. Thiele and R. H. French, Adv. Mater. 10, 1271 (1998).

${ }^{13}$ E. S. Thiele, Ph.D. thesis, University of Pennsylvania, 1998.

${ }^{14}$ The MIETAB program was obtained from A. Miller (amiller@nmsu.edu).

${ }^{15}$ L. E. McNeil and R. H. French, J. Appl. Phys. 89, 283 (2001). 